Article

\title{
In-Ear $\mathrm{SpO}_{2}$ : A Tool for Wearable, Unobtrusive Monitoring of Core Blood Oxygen Saturation
}

\author{
Harry J. Davies ${ }^{1,2, *(\mathbb{D} \text {, Ian Williams }}{ }^{1,2} \mathbb{D}$, Nicholas S. Peters ${ }^{2,3}$ and Danilo P. Mandic ${ }^{1,2}$ (D) \\ 1 Department of Electrical and Electronic Engineering, Imperial College London, London SW7 2AZ, UK; \\ i.williams10@imperial.ac.uk (I.W.); d.mandic@imperial.ac.uk (D.P.M.) \\ 2 Imperial Centre for Cardiac Engineering, Imperial College London, London SW7 2AZ, UK; \\ n.peters@imperial.ac.uk \\ 3 National Heart and Lung Institute, Faculty of Medicine, Imperial College London, London SW3 6LY, UK \\ * Correspondence: harry.davies14@imperial.ac.uk
}

Received: 5 August 2020; Accepted: 24 August 2020; Published: 28 August 2020

\begin{abstract}
The non-invasive estimation of blood oxygen saturation $\left(\mathrm{SpO}_{2}\right)$ by pulse oximetry is of vital importance clinically, from the detection of sleep apnea to the recent ambulatory monitoring of hypoxemia in the delayed post-infective phase of COVID-19. In this proof of concept study, we set out to establish the feasibility of $\mathrm{SpO}_{2}$ measurement from the ear canal as a convenient site for long term monitoring, and perform a comprehensive comparison with the right index finger-the conventional clinical measurement site. During resting blood oxygen saturation estimation, we found a root mean square difference of $1.47 \%$ between the two measurement sites, with a mean difference of $0.23 \%$ higher $\mathrm{SpO}_{2}$ in the right ear canal. Using breath holds, we observe the known phenomena of time delay between central circulation and peripheral circulation with a mean delay between the ear and finger of $12.4 \mathrm{~s}$ across all subjects. Furthermore, we document the lower photoplethysmogram amplitude from the ear canal and suggest ways to mitigate this issue. In conjunction with the well-known robustness to temperature induced vasoconstriction, this makes conclusive evidence for in-ear $\mathrm{SpO}_{2}$ monitoring being both convenient and superior to conventional finger measurement for continuous non-intrusive monitoring in both clinical and everyday-life settings.
\end{abstract}

Keywords: $\mathrm{SpO}_{2}$; hearables; wearable health; hypoxia; sleep apnea; covid-19

\section{Introduction}

One of the major roles of blood is to supply oxygen to tissues throughout the body. This is achieved through the protein haemoglobin within red blood cells, which has a high affinity to oxygen. Thus, as blood passes through capillaries in the lungs, the haemoglobin in red blood cells binds to oxygen which is subsequently pumped through arteries via the heart and transported to various tissues. The maintenance of a high arterial blood oxygen saturation is therefore extremely important, as otherwise tissues cease to be adequately supplied. The term blood oxygen saturation specifically refers to the proportion of haemoglobin in the blood that is carrying oxygen [1], and is given by

$$
\text { Oxygen Saturation }=\frac{\mathrm{HbO}_{2}}{\mathrm{HbO}_{2}+\mathrm{Hb}^{\prime}}
$$

where $\mathrm{Hb}$ refers to haemoglobin not bound with oxygen and $\mathrm{HbO}_{2}$ refers to haemoglobin bound to oxygen.

Arterial blood oxygen saturation is typically measured using pulse oximetry which gives a percentage estimate $\left(\mathrm{SpO}_{2}\right)$. It has been established that those with a healthy respiratory system typically exhibit $\mathrm{SpO}_{2}$ values of $96-98 \%$ at sea level [2]. According to the World Health Organisation, 
hypoxia is defined as a blood oxygen saturation level of less than $94 \%$, while a blood oxygen level of less than $90 \%$ may indicate the need for clinical action [3]. Hypoxic $\mathrm{SpO}_{2}$ readings are a sign of hypoxia without breathlessness in COVID-19 patients [4,5] where the major respiratory failure peaks 10 days after initial infection [6]. Indeed, in the case of COVID-19 it is strongly recommended that patients receive supplemental oxygen if their $\mathrm{SpO}_{2}$ reading falls below 90\% [7]. Hypoxic $\mathrm{SpO}_{2}$ levels may also occur in other examples of respiratory failure [8] and during breathing obstruction which is common in sleep apnea [9].

In practice, the $\mathrm{SpO}_{2}$ levels are calculated indirectly, through photoplethysmography (PPG), the non-invasive measurement of light absorption (usually red and infrared) through the blood. In short, when more blood is present, less light is reflected, so given a pulsatile increase in blood volume with each heart beat, PPG effectively measures the pulse. Depending on the level of blood oxygen saturation, the PPG measurements also experience a change in the ratio of light absorbance between the red and infrared light. Namely, the extinction coefficient of oxygenated haemoglobin with red light $(\approx 660 \mathrm{~nm})$ is lower than it is for deoxygenated haemoglobin, and the reverse is true for infrared light $(\approx 880-940 \mathrm{~nm})$ [10]. Thus, a simultaneous measurement of the absorbance of both infrared and red light allows for an estimation of blood oxygen saturation. Calculation of blood oxygen saturation, referred to as the 'ratio of ratios' method, will be fully explained in the Methods section.

Reflective PPG can be measured from any site with skin that has vasculature [11], but is, for convenience, commonly measured from the fingers (usually the index finger), wrist and earlobe. This convenience comes with some disadvantages pertaining to $24 / 7$ continuous measurements, which include the intrusive nature of measurements given that living life with a finger or earlobe clip is problematic, and also poor sensor skin contact and motion artefacts in the case of the wrist.

Other devices such as wearing a helmet for behind the ear [12] or a headband for the forehead [13], whilst providing good quality signal, giving a proxy for core blood oxygen rather than peripheral and being less obstructive to daily life, are less suitable for $24 / 7$ use as they can be perceived as stigmatising which decreases adherence to wearing the device in the patient and consumer populations.

On the other hand, the recent interest in the development of Hearables [14], has promoted the ear canal as a preferred site for the measurement of vital signs in eHealth technology. Indeed, the ear canal represents a unique opportunity for physiological measurements, due to its proximity to the brain (ear-EEG [15]), the property of the ear canal to act as a shield from external electrical noise (nature-built Faraday cage [14]), and the general fixed position of the head wrt. vital signs and neural function, as the head does not move much in daily life unlike sites such as the wrist. Owing to these desirable properties, the ear-canal has been established as a feasible wearable site by Ear-EEG $[15,16]$ and Ear-ECG [14].

When it comes to ear-PPG, unlike the finger PPG signal and the earlobe signal, it has been shown that the ear canal offers a photoplethysmogram which is stable and resistant to changes in blood volume which occur during hypothermia [17]. This is because peripheral areas of the body experience restricted blood flow during the cold, whereas the ear canal, being a narrow cavity surrounded by skin, maintains internal blood flow levels. Additionally, this makes the ear canal a preferred site for accurate core body temperature measurement. The PPG from the ear canal has also been shown to be far more sensitive than earlobe and finger PPG to amplitude variations that arise from respiration, thus allowing for a better measurement of respiration rate [18]. Furthermore, a significant delay has been observed between earlobe/behind-the-ear pulse oximetry, and pulse oximetry on the hand or the foot for detection of hypoxemia (low levels of blood oxygen) $[12,19]$, which is primarily caused by the distance from the core blood supply. Although never previously shown, a similar fast response time was hypothesised from the ear canal given that its vasculature is supplied by the carotid artery, as is the brain. The robustness of the PPG signal from the ear canal, the faster expected $\mathrm{SpO}_{2}$ response time to changes in blood oxygen levels, the opportunity for a proxy to brain oxygen saturation levels and the potential for relatively non-intrusive $24 / 7$ ambulatory monitoring of patients with breathing disorders form the motivation for our research into ear canal pulse oximetry. The aim of this study 
is therefore to investigate the feasibility, establish technical characteristics, and identify advantages in the recording of $\mathrm{SpO}_{2}$ from the ear canal, compared with the most commonly used site, the right index finger.

\section{Materials and Methods}

\subsection{Hardware}

The photoplethysmography sensor used was the MAX30101 digital PPG chip by Maxim Integrated (San Jose, CA, USA), consisting of green $(537 \mathrm{~nm})$, red $(660 \mathrm{~nm})$ and infrared $(880 \mathrm{~nm})$ light emitting diodes as well as a photo-diode to measure the reflected light. In our study, the PPG sensor was embedded in a cut out rectangular section of a viscoelastic foam earbud [20], allowing for comfortable insertion. The three light wavelengths (at $537 \mathrm{~nm}, 660 \mathrm{~nm}, 880 \mathrm{~nm}$ ) gave three measures of pulse, and also the signals required to estimate blood oxygen saturation and respiration rate. The PPG circuitry, including the MAX30101 chip, decoupling capacitors and level shifting circuitry that enable digital communication between $1.8 \mathrm{~V}$ and $3 \mathrm{~V}$ domains, was packaged in a small 3D printed case which was positioned just outside the ear canal, as shown in Figure 1. This same sensor was secured to the right index finger with medical tape for the simultaneous finger PPG measurement. The PPG chip has dimensions of $5.6 \mathrm{~mm} \times 3.3 \mathrm{~mm} \times 1.55 \mathrm{~mm}$, with the entire in-ear "Hearables" sensor having dimensions of $19 \mathrm{~mm} \times 10 \mathrm{~mm} \times 7 \mathrm{~mm}$. The width and depth of $10 \mathrm{~mm} \times 7 \mathrm{~mm}$ correspond to the viscoelastic foam earbud, which is capable of compressing to fit the ear canal.
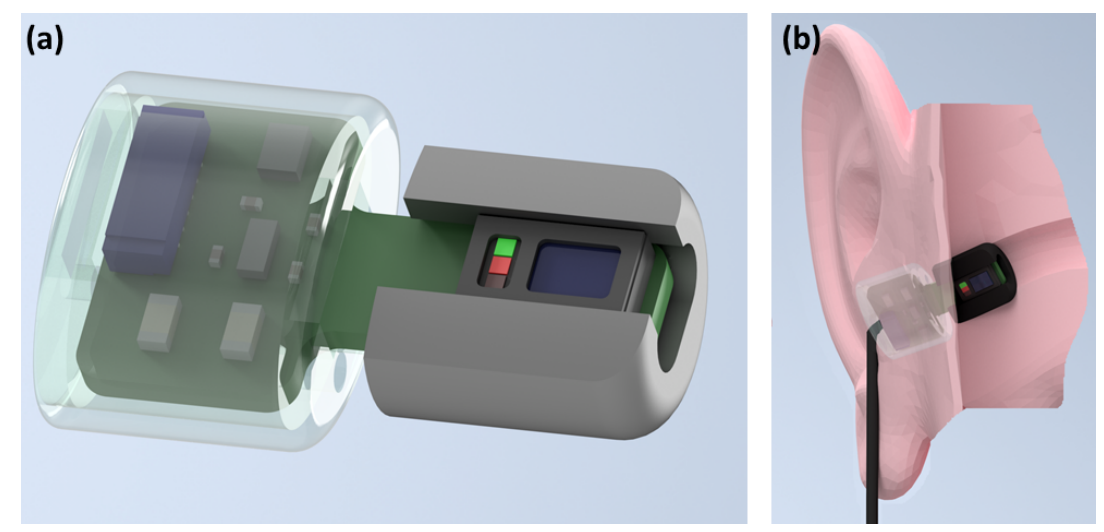

Figure 1. The in-ear photoplethysmography sensor used in our study. (a) Wearable in-ear photoplethysmography sensor for pulse oximetry, with a trimmed memory foam earbud and a 3D printed case to house the circuitry. (b) The sensor placed within the ear canal.

\subsection{Experimental Protocol}

The participants in the recordings were 14 healthy subjects ( 7 males, 7 females) aged 19-38 years. Two PPG sensors were used per subject, one secured within the right ear canal and the other to the right index finger; both sensors recorded simultaneously. The subjects were seated in front of a monitor during the recording where a video guided them on when to breathe normally, as well as when to exhale and hold their breath. The position of the sensors, monitor and recording device relative to the subject is shown in Figure 2. This video included a built in countdown, so that at every stage of the protocol, depicted in Figure 3, the subject knew the amount of time left. The experiment lasted for $435 \mathrm{~s}$, consisting of $120 \mathrm{~s}$ of normal breathing, 3 repeats of $5 \mathrm{~s}$ of exhaling and $20 \mathrm{~s}$ of breath holding with $60 \mathrm{~s}$ of normal breathing between, and a final $120 \mathrm{~s}$ of normal breathing, as depicted in Figure 3 . The exhale before the breath hold was included because if the oxygen from the lungs is expelled before holding one's breath, there is a sharper desaturation in blood oxygen than if a subject did not exhale first, thus allowing for clearer comparisons of sensitivity between the different measurement sites. Furthermore, in a real world scenario, such as obstructive sleep apnea, the lungs would not be filled with oxygen before the obstruction to inspiration occurred, making the conventional breath hold, 
where a subject inhales before holding their breath, less realistic. Subjects were also told to hold down a button, connected to the circuit board, from the moment they started exhaling until they stopped the breath hold. This was necessary, in order to know precisely when the subject was holding their breath, as reaction times would add uncertainty if the instructional display was used as the ground truth. The recordings were performed under the 881/MODREC/18 ethics approval, and all subjects gave full informed consent.

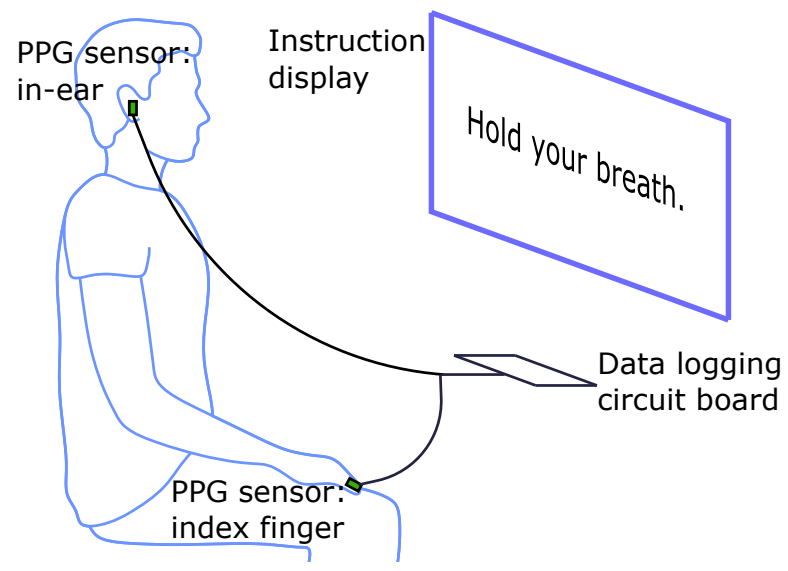

Figure 2. Simultaneous photoplethysmography (PPG) recording from the ear canal and finger. One PPG sensor is placed in the right ear canal, and another is attached to the right index finger. The simultaneous recordings are linked via a circuit board which logs the respective data streams on SD cards. The subjects are instructed on when to breathe normally, exhale and hold their breath via a video played on a monitor (see Figure 3 for the protocol), with a built in countdown to reflect the elapsed time in each phase.

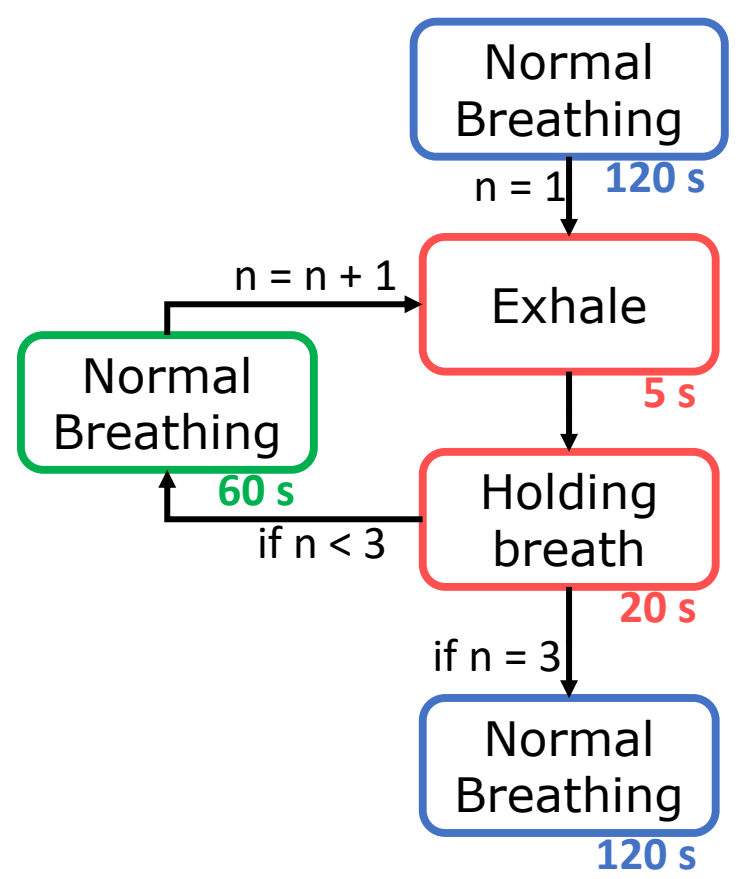

Figure 3. Flow diagram of the experiment, outlining the sequence of participants' breathing. The duration of each stage is provided beneath in seconds. The exhale and breath holding stages are repeated three times, the $60 \mathrm{~s}$ normal breathing is repeated twice, and $120 \mathrm{~s}$ of normal breathing occurs both at the start and at the end of the experiment. 


\subsection{Extraction of the $\mathrm{SpO}_{2}$ Signal}

The ratio of absorbance of infra-red to red light within the PPG sensor changes depending on the proportion of haemoglobin that is oxygenated in the blood. This change can be quantified through the so called ratio of ratios metric [21], given by

$$
R=\frac{\frac{A C_{\text {red }}}{D C_{\text {red }}}}{A C_{\text {infrared }}} .
$$

An empirically derived linear approximation can then be used to calculate an $\mathrm{SpO}_{2}$ value as a proxy to oxygen saturation. Using the manufacturer's suggested calibration [22], the $\mathrm{SpO}_{2}$ value was calculated as

$$
S p \mathrm{O}_{2}=104-17 R
$$

To obtain the alternating current (AC) components within the PPG measurements, the raw signals were firstly band-pass filtered between $1 \mathrm{~Hz}$ and $30 \mathrm{~Hz}$. Peak detection was then performed on the infrared and red AC filtered signals to find their peaks and troughs; this was achieved using the inbuilt MATLAB by MathWorks (Natick, MA, USA) function findpeaks, with a minimum peak prominence of 150 arbitrary units for the infrared signal and 30 arbitrary units for the red signal. The same procedure repeated on the same signals, but scaled by -1 , to find the troughs. Next, the peak values and trough values were separated and interpolated, before their absolute values were added together to give a constant estimate of the AC amplitude. The direct current (DC) components were obtained by low-pass filtering the raw signals at $0.01 \mathrm{~Hz}$. These waveforms are shown in Figure 4.
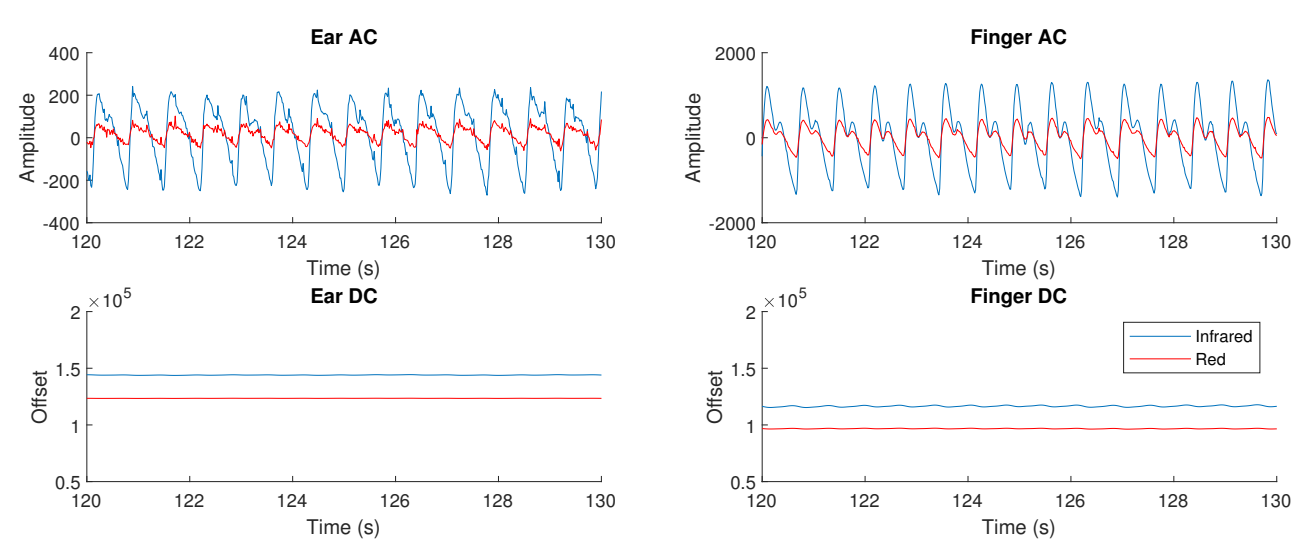

Figure 4. Examples of the alternating current (AC) and direct current (DC) waveforms within a PPG measurement, from both the right ear canal and the right index finger. The amplitude is lower in the right ear canal for both the red and infrared signals, but the signal is clear and usable. The maximum to minimum amplitude was designated as the AC signal amplitude, which is divided by the DC signal in the ratio of ratios metric to calculate $\mathrm{SpO}_{2}$.

\subsection{Data Analysis}

\subsubsection{Resting $\mathrm{SpO}_{2}$ Comparison}

The resting $\mathrm{SpO}_{2}$ was calculated over the $60 \mathrm{~s}$ section of normal breathing before the first exhale began. This time was ascertained from the timing of the first button press, explained in the Experimental Protocol subsection. The $\mathrm{SpO}_{2}$ signal from the finger and ear were averaged individually over this $60 \mathrm{~s}$ window to give a resting blood oxygen comparison for each of the measuring sites for every subject. The root mean square difference in average resting $\mathrm{SpO}_{2}$ was then calculated across all subjects, as well as the mean difference across all subjects. 


\subsection{2. $\mathrm{SpO}_{2}$ Delay}

The blood oxygen estimation delay was calculated using the button release point as the marker for minimal blood oxygen, corresponding to the point at which the breath hold ends. The time between this point of minimal blood oxygen and the first trough of the $\mathrm{SpO}_{2}$ waveform for the ear and the finger was then used to calculate the $\mathrm{SpO}_{2}$ delay for the ear, the finger and then the relative delay between the ear and the finger. Three measurements for delay were taken for each measuring site, one for every consecutive breath hold as shown in Figure 5. The mean of these three recorded delays was taken to give an average delay for each measurement site for each subject. The range, on a per subject basis, was taken as a measure of variability within the recordings for each subject. The distribution of mean delays was analysed against age and sex, whereby a paired sample $t$-test was employed to compare male and female delays while Pearson's correlation coefficient was utilised to determine if subject age was correlated to delay.

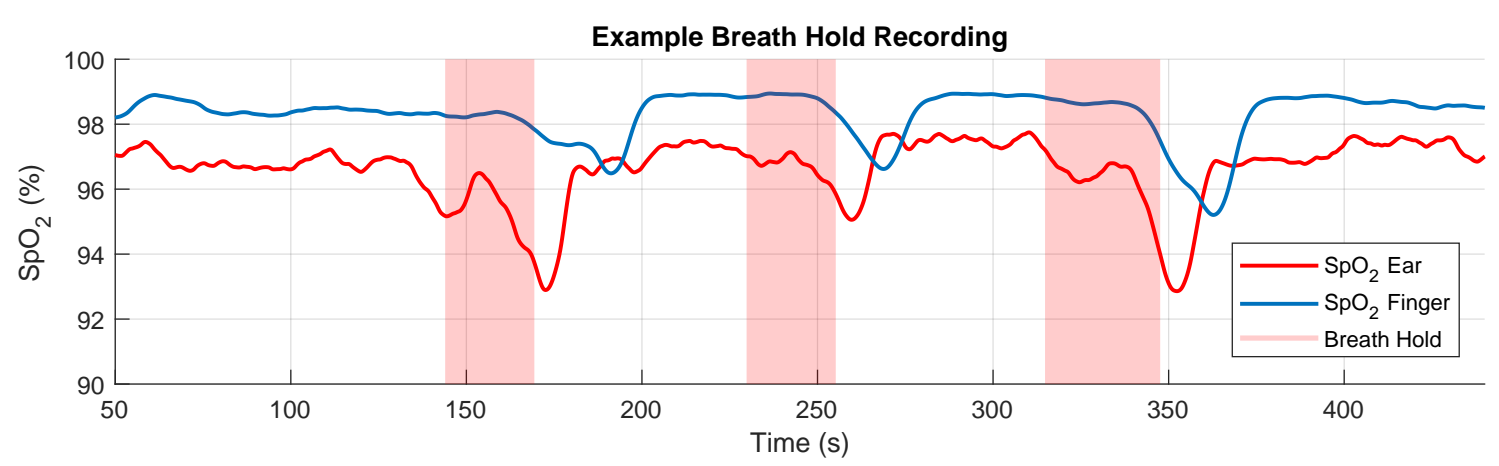

Figure 5. Exemplar $\mathrm{SpO}_{2}$ recording following the breath hold protocol (see Figure 3). The breath holds (starting with the exhale and finishing at the end of the breath hold) are designated with the shaded regions. The $\mathrm{SpO}_{2}$ recorded from the right index finger is denoted by the solid blue line, and the $\mathrm{SpO}_{2}$ recorded from the right ear canal by the solid red line. The three significant drops in $\mathrm{SpO}_{2}$ levels correspond to the three breath holds. The breath holds also vary slightly in length as they adhere to when the subject holds down a button rather than the set time of the instructional display, thus mirroring the true breath hold duration more accurately.

\section{Results}

For rigour, and to demonstrate non-inferiority of in-ear pulse oximetry to finger pulse oximetry, the experimental results span three major aspects related to the feasibility of in-ear PPG; these are the comparison of resting oxygen levels against standard finger PPG, the respective delays in detection of hypoxic events and the corresponding PPG signal amplitudes.

\subsection{Resting Oxygen Comparison}

The difference in resting $\mathrm{SpO}_{2}$ between the right ear canal and the right index finger across all subjects had a root mean square value of $1.47 \%$ with a mean difference of $0.23 \%$ higher saturation in the ear. The distribution of resting values across subjects is provided in Figure 6, and shows that all resting $\mathrm{SpO}_{2}$ values were within the physiologically healthy range of $94-100 \%$, and therefore there was a complete agreement between the ear canal and finger as measuring sites on whether or not a subject had 'healthy' blood oxygen levels. 


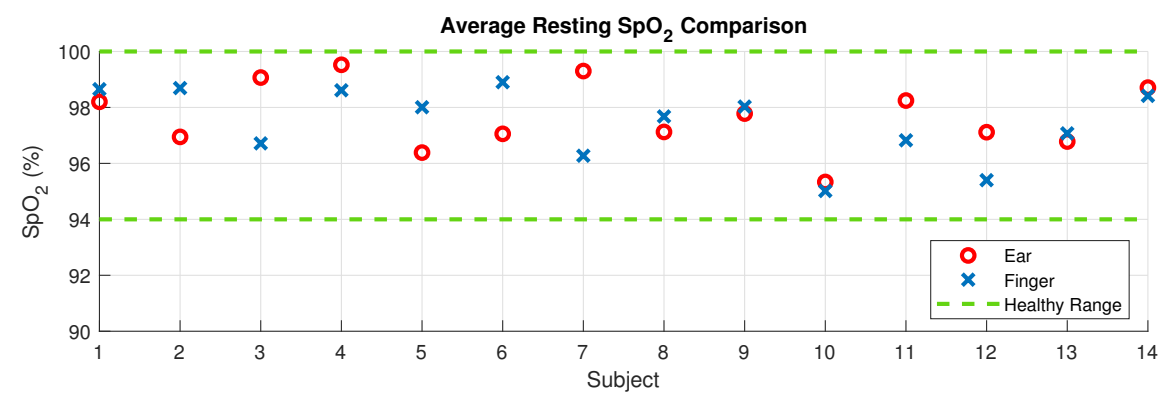

Figure 6. Mean resting $\mathrm{SpO}_{2}$ levels taken for each individual subject for the right ear canal (red circles) and the right index finger (blue crosses). The mean was taken across the 60 seconds of $\mathrm{SpO}_{2}$ data before the first breath hold sequence. The area within the two green dotted lines represents the healthy blood oxygen range of $94-100 \%$.

\subsection{Blood Oxygen Delay}

The mean relative time delay per subject between the ear and finger pulse oximetry, in other words the time it took from detecting minimal blood oxygen in the ear to detecting minimal blood oxygen in the finger, ranged from $4.18 \mathrm{~s}$ to $24.2 \mathrm{~s}$. The mean relative delay across all 14 subjects was $12.4 \mathrm{~s}$, with a mean relative delay of 9.67 seconds for the female subjects and a mean relative delay of $15.13 \mathrm{~s}$ for the male subjects. The mean female relative delay was significantly lower than the mean male relative delay $(P=0.03)$. The $\mathrm{SpO}_{2}$ delay values for all subjects are shown in Figure 7 , highlighting the large inter-subject variability in $\mathrm{SpO}_{2}$ delay and moreover the large inter-trial variability in the ear canal vs finger delay for many individual subjects.

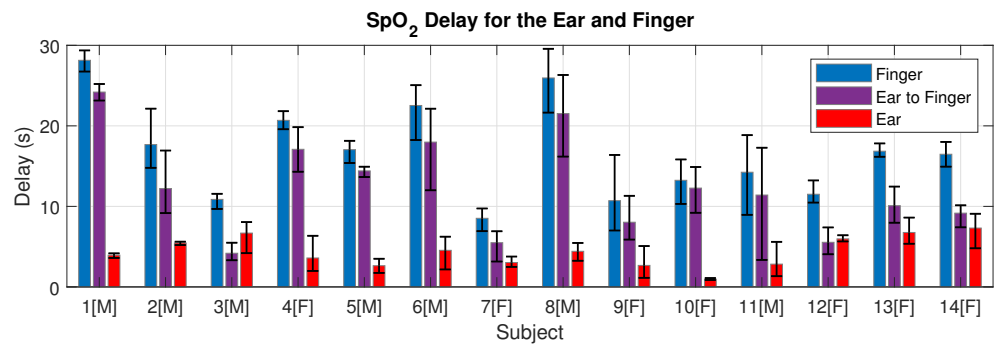

Figure 7. The absolute blood oxygen delay from the button press to the finger (blue), to the ear (red) and the relative delay between the ear and the finger (purple), shown for every subject. Male subjects are designated with $[\mathrm{M}]$ and female subjects with $[\mathrm{F}]$. The delay is calculated from the time that the breath hold stops (true minimum blood oxygen) to the time at which the measuring site detects a minimum in $\mathrm{SpO}_{2}$. Error bars are included to show the range of the three delay measurements for individual subjects.

Across our 14 subjects there was no overlap between the absolute $\mathrm{SpO}_{2}$ delay from the right ear canal and the right index finger, as shown in Figure 8. The absolute delay from the right ear canal had a mean of $4.35 \mathrm{~s}$, with a range of $0.97-7.31 \mathrm{~s}$. The absolute delay from the right index finger had a mean of $16.75 \mathrm{~s}$, with a range of $8.52-28.14 \mathrm{~s}$. When separated by sex, the mean absolute delay from the finger was $14.0 \mathrm{~s}$ for the female subjects and $19.5 \mathrm{~s}$ for the male subjects $(P=0.03)$. The mean absolute delay from the ear canal was $4.3 \mathrm{~s}$ for females and $4.4 \mathrm{~s}$ for males, as summarised in Table 1. Furthermore, there was no correlation found between age and oxygen delay across the participants. 


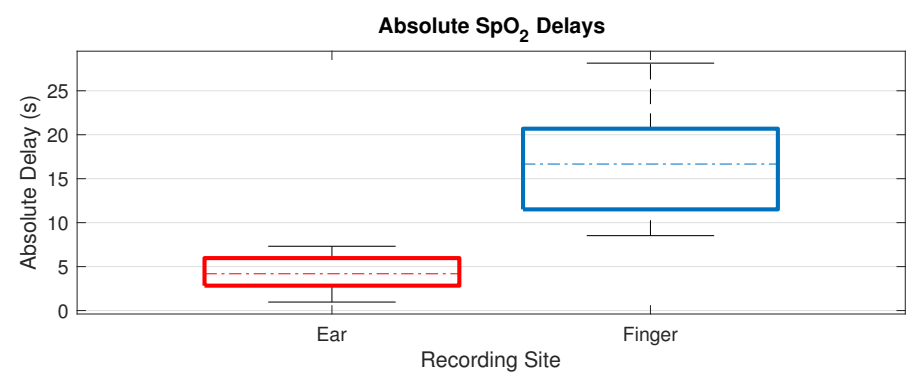

Figure 8. Boxplots of the mean absolute delays, for all subjects, between the right ear canal and the right index finger. The top and bottom of each coloured box represent respectively the upper and lower quartiles, the dotted lines represent the median, and the whisker lines extending out of the box represent the range. In our data, there is no overlap between the range of absolute delay from the ear canal and the absolute delay from the right index finger.

Table 1. Summary of mean ( \pm standard deviation) $\mathrm{SpO}_{2}$ delay values (seconds).

\begin{tabular}{cccc}
\hline & Relative & Finger & Ear Canal \\
\hline Female & $9.70 \pm 4.07$ & $14.00 \pm 4.25$ & $4.33 \pm 2.37$ \\
Male & $15.13 \pm 6.76$ & $19.49 \pm 6.28$ & $4.36 \pm 1.42$ \\
Total & $12.40 \pm 6.06$ & $16.75 \pm 5.88$ & $4.35 \pm 1.88$ \\
\hline
\end{tabular}

For rigour, the $\mathrm{SpO}_{2}$ delay between the left and right ear canal was also examined for a single subject, giving a mean delay of $0.46 \mathrm{~s}$ from the left ear canal to the right ear canal across the three breath holds, suggesting that measuring $\mathrm{SpO}_{2}$ from the left ear would yield a similar response time to the right ear, if not slightly faster.

\subsection{Photoplethysmogram Amplitude}

It was observed that 13 out of 14 subjects had a higher AC PPG amplitude from the right index finger than the right ear canal with the mean finger PPG amplitude 2.35 times higher than for the ear canal. Figure 9 presents the individual normalised resting infrared PPG amplitude for the finger and ear canal results for all 14 subjects. In the case of finger amplitude, males had a mean finger amplitude of $1.28 \times 10^{3}$ with a standard deviation of $0.76 \times 10^{3}$, compared with females who had a mean finger amplitude of $0.90 \times 10^{3}$ and a standard deviation of $0.49 \times 10^{3}$. In the case of ear amplitude, males had a mean ear amplitude of $0.59 \times 10^{3}$ with a standard deviation of $0.40 \times 10^{3}$, compared with females that had a mean finger amplitude of $0.33 \times 10^{3}$ and a standard deviation of $0.12 \times 10^{3}$. Neither sex difference between finger amplitudes and ear amplitudes was statistically significant, with $P=0.14$ and $P=0.05$ respectively. Importantly, even in the lowest amplitude cases, the in-ear PPG signals were effective for peak detection and thus usable for the calculation of $\mathrm{SpO}_{2}$.

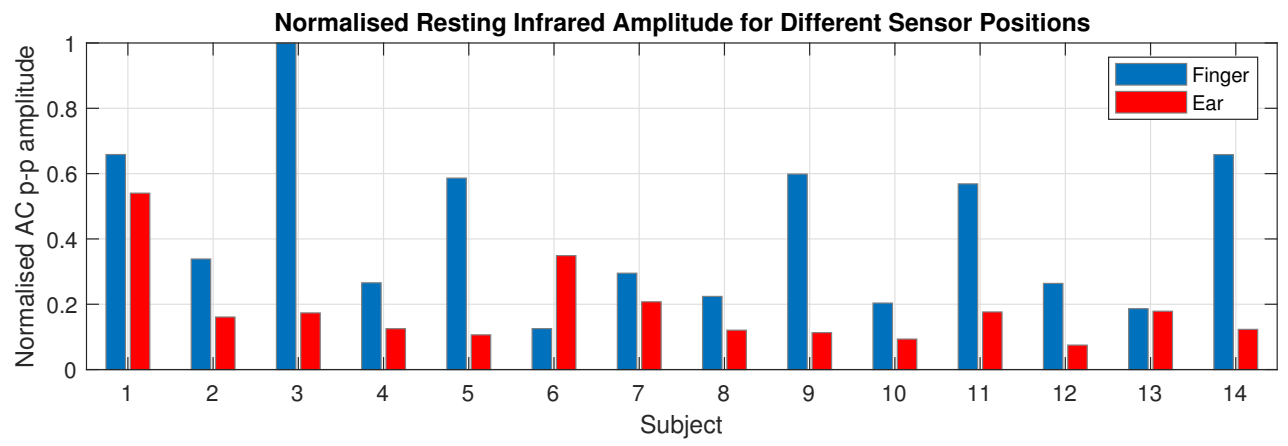

Figure 9. The mean resting maximum to minimum AC amplitude of the photoplethysmography signal for all subjects. These values were measured from the $60 \mathrm{~s}$ of filtered PPG data before the first breath hold and were normalised to the maximum mean AC amplitude across all subjects. 


\section{Discussion}

\subsection{Resting $\mathrm{SpO}_{2}$}

Blood oxygen saturation measurements from the right ear canal and the right index finger were comparable at rest, with a minimal systematic offset between the two, indicated by the mean difference of $0.23 \%$ higher resting $\mathrm{SpO}_{2}$ in the right ear canal, as shown in Figure 6. The variability between the two measurement sites, indicated by the root mean square difference of $1.47 \%$, is to be expected, given that mean $\mathrm{SpO}_{2}$ values were shown to vary as much as $0.9 \%$ even across fingers on the same hand [23]. The blood oxygen saturation delay between the ear and the finger may also be a source of error when using the same absolute $60 \mathrm{~s}$ time window for comparison, although given that the window was long in comparison with the $\mathrm{SpO}_{2}$ delay and the fact that resting values stay fairly constant, this source of error should be minimal. Both sensor positions were in $100 \%$ agreement concerning the patients having healthy $\mathrm{SpO}_{2}$ levels of 94 to $100 \%$. The established similarity in the resting oxygen saturation values with the commonly used right index finger location are a first step towards validating the ear canal as an alternative measurement site for pulse oximetry.

\section{2. $\mathrm{SpO}_{2}$ Delay}

Our results indicate a mean oxygen saturation delay between the right ear canal and the right index finger of $12.4 \mathrm{~s}$. In other words, a steep drop in $\mathrm{SpO}_{2}$ is detected on average $12.4 \mathrm{~s}$ faster from the right ear canal than it is from the right index finger, with two subjects exhibiting over $20 \mathrm{~s}$ faster detection from the right ear canal. The faster response from the ear-canal strongly recommends the incorporation of in-ear $\mathrm{SpO}_{2}$ measurements into so-called Hearables, as there are many situations where fast $\mathrm{SpO}_{2}$ response time is critical, such as improving outcomes during neonatal resuscitation [24] and closed loop automatic oxygen therapy [25]. Moreover, this opens the avenue to aid comparison of sleep stage scoring and sleep apnea events by aligning changes in $\mathrm{SpO}_{2}$ to the correct $30 \mathrm{~s}$ sleep epoch [26].

We found a large inter-subject variability in both the absolute oxygen delay to the right ear canal and the absolute oxygen delay to the right index finger. On average, the female subjects had lower relative oxygen delay between the ear and the finger, caused by the much smaller absolute delay to the right index finger in combination with an almost identical absolute delay to the right ear canal. We hypothesise that this could be related to males being taller on average and therefore having longer arms, which would give oxygenated blood further to travel from the heart to the fingers. Variations in height and arm length may also explain some of the large inter-subject variability in absolute $\mathrm{SpO}_{2}$ delays. With a taller subject we would expect on average a longer distance for blood to travel to both the ear canal and the fingers, but we found no correlation between the absolute delay to the ear and absolute delay to the finger. One relevant factor was average heart rate across the trials, which we found to have a significant moderate positive correlation with absolute delay to the ear $(R=0.56$, $P=0.03$ ), but was not correlated with absolute delay to the finger. Importantly, age was not found to be a correlating factor to oxygen delay in our subjects. Future work must consist of investigating the efficacy of in-ear $\mathrm{SpO}_{2}$ as well as the $\mathrm{SpO}_{2}$ delay in older cohorts that may have underlying respiratory conditions, and not just young healthy subjects. The natural question arises, could $\mathrm{SpO}_{2}$ delay be an indicator of the health of a patients circulatory system?

Whilst not as precise as arterial blood gas analysis, this is the first attempt to measure the blood oxygen delay to the ear itself. Importantly it should be noted that although the ear canal responds faster to changes in blood oxygen and much more closely resembles core blood oxygen than peripheral sites such as the finger, it is still not instantaneous, with our results showing a mean blood oxygen delay to the ear of $4.35 \mathrm{~s}$.

\subsection{Photoplethysmogram Amplitude}

The in-ear PPG in this study was found to have consistently smaller amplitudes from the ear canal when compared with the finger. The three reasons for a lower PPG amplitude are: (1) less vascular 
density of the tissue (2) poorer sensor contact with the skin and (3) arterial pulse pressure amplitude. Considering that the ear and fingers are both common PPG placement sites, given the high density of arterioles and capillaries $[27,28]$, the most likely explanations for a lower PPG amplitude are therefore sensor placement issues within the ear canal and a lower carotid arterial pulse amplitude compared to the radial artery in the arm.

The large variability in ear canal sizes was somewhat mitigated by different available earbud sizes, but even with the smallest earbud some subjects found it difficult to insert the sensor fully into the ear canal. Some subjects with wider ear canals also noted that the sensor became looser during the trial. These issues did not affect the conclusive nature of this feasibility study and will be addressed in future with an improved sensor design, such as by employing ear-hooks, commonly used with sports headphones, to stabilise the position of sensor within the ear.

Even disregarding placement issues, a lower PPG pulse magnitude may be expected from the ear canal given a higher pressure wave amplitude in the radial artery of the wrist leading to the fingers, compared to the carotid artery leading to the ear canal [29]. This is also evidenced by the broader peak from the ear canal, shown in Figure 4, which is a characteristic of the pressure wave found in the carotid artery.

Importantly, despite a lower signal amplitude, all data recorded from the ear canal was functional for peak detection of the AC PPG signal and therefore the $\mathrm{SpO}_{2}$ calculation.

\section{Conclusions}

There is a growing need for improved non-intrusive wearable $\mathrm{SpO}_{2}$ recording across many different scenarios, from the monitoring of sleep apnea to the tracking of vital signs of outpatients with a threat of respiratory deterioration, such as those with general breathing disorders or those recovering from COVID-19. To this end, we have investigated the possibility of estimating blood oxygen saturation from the ear canal in healthy subjects and have comprehensively demonstrated its non-inferiority compared with conventionally used finger pulse oximetry, with a significantly faster response time averaging $12.4 \mathrm{~s}$. The results have indicated that the favourable speed of in-ear pulse oximetry, in conjunction with the previously documented advantage of resilience to changes in circulation associated with environmental temperature changes (summarised in Table 2), may offer significant clinical advantages and a proxy to brain oxygen levels. Given its privileged position on the human body and a fixed distance to vital signs during most daily activities and while sleeping, the ear canal may even be a superior site for measurement of $\mathrm{SpO}_{2}$ in the scenario of $24 / 7$ continuous monitoring. In an era of urgency for enhanced tracking of hypoxia and increasing use of smart devices for health monitoring, this proof of concept study provides a compelling argument for the integration of ear canal pulse oximetry into current state-of-the-art Hearables [14].

Table 2. Summary of different $\mathrm{SpO}_{2}$ sensor positions.

\begin{tabular}{ccc}
\hline Placement & Fast Response & Robust to Temperature \\
\hline Finger & No & No \\
Earlobe & Yes [19] & No \\
Ear canal & Yes & Yes [17] \\
\hline
\end{tabular}

Author Contributions: Conceptualization, H.J.D. and D.P.M.; methodology, H.J.D.; software, H.J.D. and I.W.; validation, H.J.D., D.P.M. and N.S.P.; formal analysis, H.J.D.; investigation, H.J.D.; resources, I.W., H.J.D. and D.P.M.; data curation, H.J.D.; writing—original draft preparation, H.J.D., D.P.M., N.S.P. and I.W.; writing一review and editing, H.J.D., D.P.M. and N.S.P.; visualization, H.J.D.; supervision, D.P.M. and N.S.P.; project administration, D.P.M.; funding acquisition, D.P.M. and N.S.P. All authors have read and agreed to the published version of the manuscript.

Funding: This work was supported by the Racing Foundation grant 285/2018, MURI/EPSRC grant EP/P008461, the Dementia Research Institute at Imperial College London, the BHF Centre of Research Excellence, the Imperial Centre for Cardiac Engineering, the Imperial Health Charity and the NIHR Biomedical Research Centre. 
Conflicts of Interest: The authors declare no conflict of interest.

\section{References}

1. Jubran, A. Pulse oximetry. Crit. Care 1999, 3, R11. [CrossRef] [CrossRef] [PubMed]

2. Smith, G.B.; Prytherch, D.R.; Watson, D.; Forde, V.; Windsor, A.; Schmidt, P.E.; Featherstone, P.I.; Higgins, B.; Meredith, $\mathrm{P} \mathrm{SpO}_{2}$ values in acute medical admissions breathing air-Implications for the British Thoracic Society guideline for emergency oxygen use in adult patients? Resuscitation 2012, 83, 1201-1205. [CrossRef] [CrossRef]

3. World Health Organization. Pulse Oximetry Training Manual; World Health Organization: Geneva, Switzerland, 2011.

4. Gattinoni, L.; Chiumello, D.; Caironi, P.; Busana, M.; Romitti, F.; Brazzi, L.; Camporota, L. COVID-19 pneumonia: Different respiratory treatments for different phenotypes? Intensive Care Med. 2020. [CrossRef] [CrossRef]

5. Cascella, M.; Rajnik, M.; Cuomo, A.; Dulebohn, S.C.; Di Napoli, R. Features, Evaluation and Treatment Coronavirus (COVID-19); StatPearls Publishing: Treasure Island, FL, USA 2020.

6. Pan, F.; Ye, T.; Sun, P.; Gui, S.; Liang, B.; Li, L.; Zheng, D.; Wang, J.; Hesketh, R.L.; Yang, L.; et al. Time Course of Lung Changes On Chest CT During Recovery From 2019 Novel Coronavirus (COVID-19) Pneumonia. Radiology 2020, 200370. [CrossRef] [CrossRef] [PubMed]

7. Alhazzani, W.; Møller, M.H.; Arabi, Y.M.; Loeb, M.; Gong, M.N.; Fan, E.; Oczkowski, S.; Levy, M.M.; Derde, L.; Dzierba, A.; et al. Surviving Sepsis Campaign: guidelines on the management of critically ill adults with Coronavirus Disease 2019 (COVID-19). Intensive Care Med. 2020, 1-34. [CrossRef] [CrossRef] [PubMed]

8. Khemani, R.G.; Thomas, N.J.; Venkatachalam, V.; Scimeme, J.P.; Berutti, T.; Schneider, J.B.; Ross, P.A.; Willson, D.F.; Hall, M.W.; Newth, C.J.L. Comparison of $\mathrm{SpO}_{2}$ to $\mathrm{PaO}_{2}$ based markers of lung disease severity for children with acute lung injury. Critical Care Med. 2012, 40, 1309-1316. [CrossRef] [CrossRef]

9. Oksenberg, A.; Arons, E.; Nasser, K.; Vander, T.; Radwan, H. REM-related Obstructive Sleep Apnea: The Effect of Body Position. J. Clin. Sleep Med. 2010, 6, 343-348. [CrossRef] [PubMed]

10. Nitzan, M.; Romem, A.; Koppel, R. Pulse oximetry: Fundamentals and technology update. Med. Devices (Auckl. NZ) 2014, 7, 231. [CrossRef] [CrossRef]

11. Nilsson, L.; Goscinski, T.; Kalman, S.; Lindberg, L.G.; Johansson, A. Combined photoplethysmographic monitoring of respiration rate and pulse: A comparison between different measurement sites in spontaneously breathing subjects. Acta Anaesthesiol. Scand. 2007, 51, 1250-1257. [CrossRef] [CrossRef]

12. Bradke, B.; Everman, B. Investigation of Photoplethysmography Behind the Ear for Pulse Oximetry in Hypoxic Conditions with a Novel Device (SPYDR). Biosensors 2020, 10, 34. [CrossRef] [CrossRef]

13. Dresher, R. Wearable Forehead Pulse Oximetry: Minimization of Motion and Pressure Artifacts. Master's Thesis, Worcester Polytechnic Institute, Worcester, MA, USA, 2006.

14. Goverdovsky, V.; Von Rosenberg, W.; Nakamura, T.; Looney, D.; Sharp, D.J.; Papavassiliou, C.; Morrell, M.J.; Mandic, D.P. Hearables: Multimodal physiological in-ear sensing. Sci. Rep. 2017, 7, 1-10. [CrossRef] [CrossRef] [PubMed]

15. Looney, D.; Kidmose, P.; Park, C.; Ungstrup, M.; Rank, M.; Rosenkranz, K.; Mandic, D. The in-the-ear recording concept: User-centered and wearable brain monitoring. IEEE Pulse 2012, 3, 32-42. [CrossRef] [CrossRef] [PubMed]

16. Nakamura, T.; Alqurashi, Y.D.; Morrell, M.J.; Mandic, D.P. Hearables: Automatic Overnight Sleep Monitoring with Standardized In-Ear EEG Sensor. IEEE Trans. Biomed. Eng. 2020, 67, 203-212. [CrossRef] [CrossRef] [PubMed]

17. Budidha, K.; Kyriacou, P.A. Investigation of photoplethysmography and arterial blood oxygen saturation from the ear-canal and the finger under conditions of artificially induced hypothermia. In Proceedings of the IEEE Annual International Conference of the Engineering in Medicine and Biology Society, EMBS, Milan, Italy, 25-29 August 2015; pp. 7954-7957. [CrossRef]

18. Budidha, K.; Kyriacou, P.A. In vivo investigation of ear canal pulse oximetry during hypothermia. J. Clin. Monit. Comput. 2018, 32, 97-107. [CrossRef] [CrossRef]

19. Hamber, E.A.; Bailey, P.L.; James, S.W.; Wells, D.T.; Lu, J.K.; Pace, N.L. Delays in the detection of hypoxemia due to site of pulse oximetry probe placement. J. Clin. Anesth. 1999, 11, 113-118. [CrossRef] [CrossRef] 
20. Goverdovsky, V.; Looney, D.; Kidmose, P.; Mandic, D.P. In-Ear EEG from viscoelastic generic earpieces: Robust and unobtrusive 24/7 monitoring. IEEE Sens. J. 2016, 16, 271-277. [CrossRef] [CrossRef]

21. Rusch, T.L.; Sankar, R.; Scharf, J.E. Signal processing methods for pulse oximetry. Comput. Biol. Med. 1996, 26, 143-159. [CrossRef] [CrossRef]

22. MaximIntegrated. Recommended Configurations and Operating Profiles for MAX30101/MAX30102 EV Kits; Technical Report; MaximIntegrated: San Jose, CA, USA, 2018.

23. Basaranoglu, G.; Bakan, M.; Umutoglu, T.; Zengin, S.U.; Idin, K.; Salihoglu, Z. Comparison of $\mathrm{SpO}_{2}$ values from different fingers of the hands. SpringerPlus 2015, 4, 561. [CrossRef] [CrossRef]

24. Baquero, H.; Alviz, R.; Castillo, A.; Neira, F.; Sola, A. Avoiding hyperoxemia during neonatal resuscitation: Time to response of different $\mathrm{SpO}_{2}$ monitors. Acta Paediatr. 2011, 100, 515-518. [CrossRef] [CrossRef]

25. Morozoff, E.P.; Smyth, J.A. Evaluation of three automatic oxygen therapy control algorithms on ventilated low birth weight neonates. In Proceedings of the 31st IEEE Annual International Conference of the Engineering in Medicine and Biology Society, Minneapolis, MI, USA, 3-6 September 2009; pp. 3079-3082. [CrossRef]

26. Berry, R.B.; Brooks, R.; Gamaldo, C.E.; Harding, S.M.; Lloyd, R.M.; Quan, S.F.; Troester, M.M.; Vaughn, B.V. AASM I Scoring Manual Version 2.4 The AASM Manual for the Scoring of Sleep and Associated Events; American Academy of Sleep Medicine: Darien, IL, USA ; 2017.

27. Mannheimer, P.D. The Light-Tissue Interaction of Pulse Oximetry. Anesth. Analg. 2007, 105, S10-S17. [CrossRef] [CrossRef]

28. Alvord, L.S.; Farmer, B.L. Anatomy and Orientation of the Human External Ear. J.-Am. Acad. Audiol. 1997, 8, 383-390 [PubMed]

29. Kelly, R.; Hayward, C.; Avolio, A.; O’Rourke, M. Noninvasive determination of age-related changes in the human arterial pulse. Circulation 1989, 80, 1652-1659. [CrossRef] [CrossRef] [PubMed]

(C) 2019 by the authors. Licensee MDPI, Basel, Switzerland. This article is an open access article distributed under the terms and conditions of the Creative Commons Attribution (CC BY) license (http:/ / creativecommons.org/licenses/by/4.0/). 